\section{Habilidades clínicas para el manejo de trastornos depresivos en médicos generales en Santiago de Chile}

\author{
JULIA ACUÑA, KARINA RDZ-NAVARRO ${ }^{a}$, GABRIELA HUEPE ${ }^{\mathrm{b}}$, \\ ALBERTO BOTTO, MARCELO CÁRCAMO ${ }^{c}$, JUAN PABLO JIMÉNEZ
}

\section{Clinical skills of Chilean general practitioners for the management of depressive disorders}

Background: Diverse and sustained efforts have been developed to improve the management of depression by general practitioners (GPs), but they have not improved treatment coverage and quality of services. Aim: To explore the level of knowledge and clinical skills to diagnose and treat depression by GPs in Primary Health Care (PHC) in the Metropolitan Area of Santiago de Chile (RM). Material and Methods: Theoretical knowledge (TK), diagnostic skills (DS) and treatment skills (TS) were evaluated in 56 GPs of the RM with a battery of specially designed instruments. Results: In TK there were significant differences between GPs aged 31 years or less and their older counterparts and between Chilean and foreign doctors. Five percent of observed differences in $T K$ were explained by age and nationality, respectively. Chilean GPs achieved higher scores in recognition of symptoms (RS), one of the dimensions of DS. No significant differences by age or nationality were observed for DS and TS. Conclusions: Achievement of GPs on tests measuring TK, DS, and TS was generally below 50\%. This deficiency should be improved.

(Rev Med Chile 2016; 144: 47-54)

Key words: Clinical competence; Depressive disorder; General practitioners; Primary Health Care

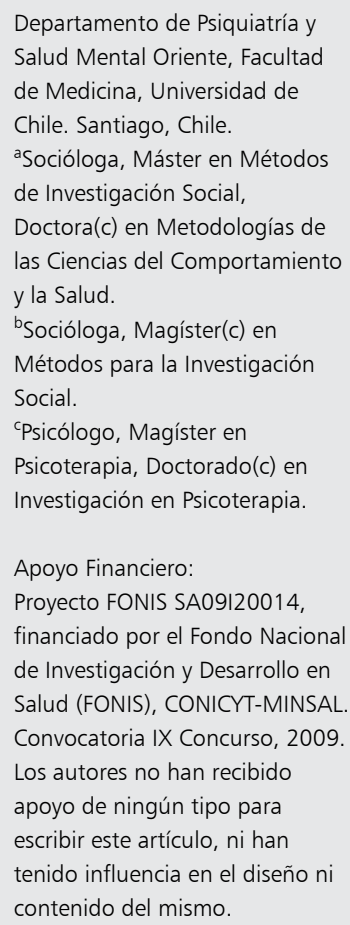

Recibido el 11 de mayo de 2015, aceptado el 9 de octubre de 2015.

Correspondencia a:

Julia Acuña R.

Av. Salvador 486, Providencia,

Santiago de Chile.

julia.acuna@gmail.com

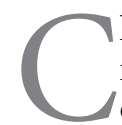

hile se encuentra entre los países con tasas más altas de patología psiquiátrica, tanto en estudios poblacionales como en consultantes en Atención Primaria de Salud (APS) ${ }^{1-6}$, lo cual se ha reflejado en las políticas públicas de salud $^{7}$. A partir de la Reforma de Salud, diferentes enfermedades mentales se han incorporado entre las Garantías Explicitas de Salud (GES) donde destaca el Programa de Tratamiento de la Depresión en $\mathrm{APS}^{8,9}$ y, desde 2007, la Guía Clínica GES Depresión $(2007,2009,2013)^{10,11}$.

Desde la década de los ochenta la OMS plantea que el nivel primario de salud constituye el eje central de los servicios sanitarios, en el cual el médico general (MG) cumple un rol fundamental. Se han desarrollado diversas estrategias para mejorar las habilidades clínicas en salud mental de los MGs en APS $^{12,13}$ siendo la capacitación una de las más utilizadas. Sin embargo, no sólo persiste una importante brecha de cobertura entre la prevalencia real y la prevalencia tratada sino también problemas en la calidad de los servicios, inclusive los servicios especializados ${ }^{14-18}$. Diversos estudios han establecido que no se ha logrado mejorar las prácticas clínicas de los MGs probablemente porque los esfuerzos se han centrado principalmente en el desarrollo de habilidades cognitivas, ${ }^{9,11,19-22}$, desatendiendo otras habilidades, creencias, actitudes y el involucramiento de los MGs en el proceso enseñanza-aprendizaje $\mathrm{e}^{23-25}$.

La educación médica durante las últimas dos décadas se ha orientado hacia la formación de 
competencias en diferentes áreas: cuidado del paciente, habilidades interpersonales, comunicacionales y conocimientos médicos. Se plantea que con ellas los MGs estarían mejor capacitados para investigar, valorar, seleccionar y tomar buenas decisiones en diferentes situaciones clínicas ${ }^{25-30}$. Si bien la formación médica de pregrado en Chile se encuentra en la etapa de implementación de un currículum basado en competencias ${ }^{31-34}$, se ha planteado que ésta resulta insuficiente en materia de psiquiatría y salud mental, ya sea por limitaciones de tiempo o por tratarse de objetivos formativos complejos que requieren metodologías de enseñanza-aprendizaje difíciles de implemen$\operatorname{tar}^{31}$; por lo tanto, como advierte Leigh y cols. ${ }^{35,36}$ se requiere de formación de postítulo en estas materias. Por ello, se observa un giro de la investigación en educación médica hacia la evaluación de conocimiento aplicado, de habilidades y actitudes en la relación clínica como componentes de la competencia profesional ${ }^{23,25}$.

Con el objeto de abordar el problema de la formación de postítulo en Depresión, se ejecutó un proyecto FONIS (SA09I20014) de investigación e intervención, enfocado en aspectos claves tanto técnicos, como cognitivos y emocionales de la práctica médica, componentes de la competencia profesional del clínico ${ }^{37}$, el cual fue aprobado por el Comité de Ética de Investigación de la Facultad de Medicina de la Universidad de Chile. El propósito fue evaluar el impacto de un nuevo modelo de capacitación en trastornos depresivos sobre las habilidades diagnósticas y terapéuticas del MG, en base a un diseño experimental randomizado que consideró tres mediciones: previo, al término y a los tres meses finalizada la capacitación ${ }^{38}$. Este artículo presenta los resultados del estudio basal, exploratorio con alcances descriptivos, en una muestra de MGs de APS de ambos sexos, de diversas nacionalidades y edades de la Región Metropolitana de Santiago (RM).

\section{Material y Métodos}

\section{Muestra}

La muestra consistió en MGs de APS de Servicios de Salud de la Región Metropolitana, de ambos sexos, con una jornada laboral mínima de $22 \mathrm{~h}$ semanales en los consultorios, reclutados de forma voluntaria a través de diferentes vías de difusión del estudio y de la capacitación ofrecida como parte de
Tabla 1. Descripción de la muestra

\begin{tabular}{|llc|}
\hline Variables & Categorías & $\%$ (n) \\
Sexo & Femenino & $67,9(38)$ \\
& Masculino & $32,1(18)$ \\
Edad & Adulto joven (menor de 31 años) & $53,6(30)$ \\
& Adulto (mayor de 31 años) & $46,6(26)$ \\
Nacionalidad & Chilenos & $42,9(24)$ \\
& Extranjeros & $57,1(32)$ \\
Experiencia & Baja (5 a 34 meses) & $51,8(29)$ \\
en APS & Media a alta (mayor a 34 meses) & $48,2(27)$ \\
Jornada en & Completa (40 a 44 h) & $91,1(51)$ \\
APS & Menos que jornada completa & $8,9(6)$ \\
\hline
\end{tabular}

él, tales como afiches, cartas, etc. Hubo 76 médicos interesados, de los cuales se seleccionaron 56 que cumplían los criterios de inclusión en el estudio. Con el fin de disponer de una muestra relativamente homogénea, se estableció como requisito de inclusión una permanencia mínima de 6 meses y una máxima de 60 meses en APS. Se excluyó a médicos que tuvieran alguna capacitación previa en salud mental. Las características de la muestra están resumidas en la Tabla 1.

\section{Variables e instrumentos de medición}

Se definieron tres variables de interés: Conocimientos Teóricos (CT), Habilidades Diagnósticas (HD) y Habilidades Terapéuticas (HT), cuyas descripciones, dimensiones e instrumentos de evaluación se resume en Tabla 2.

\section{Construcción de índices}

Para cada dimensión de interés se construyó un índice agrupando las preguntas pertinentes. Este proceso fue realizado en cuatro fases:

1. Construcción de índices sumatorios simples de respuestas correctas (ISRC), respuestas incorrectas (ISRI).

2. Cálculo de porcentajes respuestas correctas (PrC) e incorrectas respecto del total (PrI).

3. Construcción de índice global bruto (IGB) para cada dimensión, sancionando las respuestas incorrectas mediante el descuento de la probabilidad de respuesta al azar. Cada índice fue construido en base a la siguiente fórmula:

$$
I G B=\operatorname{Pr} C-\frac{\operatorname{Pr} I}{n_{k}}
$$


Tabla 2. Variables, dimensiones e instrumentos de evaluación de habilidades diagnósticas y terapéuticas para el manejo de la depresión por médicos generales de APS de RM. Santiago, Chile

Variables
Conocimientos teóricos en depresión:
Conjunto organizado de información propia de
la disciplina médica relacionada con depresión;
con ellos es posible resolver problemas o tomar
decisiones clínicas

Habilidades diagnósticas: Capacidades del MG de reconocer síntomas en el paciente y clasificarlos dentro de categorías patológicas predeterminadas de acuerdo con la clasificación internacional de enfermedades (CIE-10) para los Trastornos del Ánimo

Habilidades terapéuticas: Conjunto de decisiones que el MG toma en relación con un paciente; tienen por objetivo dar respuesta adecuada a la demanda que el paciente plantea (motivo de consulta)

\author{
Dimensiones \\ Epidemiología \\ Etiopatogenia \\ Manifestaciones clínicas \\ Proceso diagnóstico \\ Proceso terapéutico
}

Reconocimiento de síntomas: Capacidad de identificar aquellas manifestaciones de enfermedades relacionadas con trastorno del ánimo que surgen del reporte subjetivo del paciente y/o de la observación directa del médico Clasificación de síntomas: Ordenamiento de los síntomas presentes en una entrevista clínica y la decisión que hace el médico respecto de si se cumple los requisitos para plantear la presencia de un diagnóstico establecido de acuerdo a criterios diagnósticos CIE-10

Tratamiento inicial: Lugar de tratamiento, tipo de tratamiento y tratamiento farmacológico (tipo de fármaco y dosis)

Tratamiento de continuación: Lugar de tratamiento, tipo de tratamiento y tratamiento farmacológico (tipo de fármaco y dosis)

\section{Instrumentos}

Batería de 18 preguntas de alternativas con una sola respuesta correcta

Plantilla estándar de síntomas a reconocer en 3 viñetas videadas de casos clínicos reales

Pregunta estándar para 4 viñetas escritas de casos clínicos construidos

Pregunta estándar para 6 viñetas cortas escritas de casos clínicos construidos
4. Finalmente, a partir del IGB de cada dimensión y para facilitar la interpretación de los resultados, se construyó un índice estandarizado para cada dimensión (ISTD) que podía asumir valores de 0 a 100 , donde valores cercanos a 0 indicarán niveles bajos del rasgo y cercanos a 100 niveles altos del rasgo. Para construir el ISTD de cada dimensión se utilizó la puntuación teórica mínima (pt_min) y máxima (pt_max) aplicando la siguiente fórmula:

$$
I S T D=\frac{\left(I G B-p t_{-} \min \right)}{p t \max +p t \min } * 100
$$

\section{Validación de los instrumentos}

Los instrumentos generados fueron sometidos a un proceso de evaluación de su validez de constructo $^{\star}$ en tres fases:

1. Evaluación por jueces expertos: Tanto las definiciones nominales como operacionales, así como los cuestionarios fueron evaluados por tres expertos en la guía GES y en Salud Pública, con acreditada experiencia clínica en psiquiatría general, quienes sugirieron modificaciones menores a las definiciones y preguntas, las cuales fueron acogidas. Se elaboró una segunda versión de los instrumentos que fue sometida a la evaluación de un metodólogo cuantitativo con experiencia en construcción y validación de instrumentos de medición, quien añadió nuevas sugerencias que se incorporaron en la versión final.

2. Pretest: Una vez construido los instrumentos definitivos, fueron pretesteados con internos de medicina y con residentes principiantes de psiquiatría. Los resultados del pilotaje fueron analizados estadísticamente y se incorporaron nuevas modificaciones a las preguntas que presentaron un bajo grado de discriminación.

3. Piloto: Los instrumentos así modificado fueron nuevamente piloteados en un grupo de internos de medicina y con residentes principiantes de psiquiatría para garantizar su adecuado funcionamiento en la aplicación final a la muestra de MGs.

\footnotetext{
${ }^{\star}$ La versión definitiva de los instrumentos, se encuentra a disposición de los interesados previa solicitud a los autores.
} 


\section{Análisis de los datos}

Los datos fueron analizados mediante SPSS Statistics 17.0 (SPSS Inc., 2008), considerando grupos por sexo (hombre o mujer), edad (adulto joven o adulto) y nacionalidad (chilenos o extranjeros). Los resultados de los índices estandarizados fueron analizados utilizando pruebas de diferencias de medias ( $\mathrm{t}$ de student) para las variables cuantitativas y diferencias de medianas para las variables categóricas (U de Mann-Whitney), así como también estadísticos de tamaño efecto pertinentes al nivel de medición de las variables ( $\mathrm{d}$ de Cohen y estadístico A).

\section{Resultados}

\section{Conocimientos teóricos $(C T)$}

La muestra de médicos reclutada para el estudio presentó niveles "medio-bajos" de conocimientos teóricos en depresión ( $\mathrm{M}=38,9$; IC: 35,8-41,9), con valores mínimos $(\mathrm{Min}=16,7)$ y máximos $(\mathrm{Max}=61,1)$ que no comprenden los límites teóricos de la variable establecidos en 0 y 100 , presentando a su vez un grado de homogeneidad dentro de los niveles aceptables ( $\mathrm{SD}=11,4 ; \mathrm{VC}=0,29)$, lo cual indica que los casos se encuentran relativamente concentrados en torno al promedio.
Los médicos adultos jóvenes presentaron un nivel significativamente más alto de CT que médicos adultos, apreciándose que la edad explica $5,3 \%$ de las diferencias observadas en CT. El grupo de médicos chilenos mostró un nivel significativamente más alto de CT que el grupo de médicos extranjeros. Esta variable explica $4,8 \%$ de las diferencias observadas en CT. Cabe destacar, sin embargo, que quienes obtuvieron los niveles más altos en CT se encontraban bajo 50\% del índice. Esto significa que el nivel de CT observado en todo el grupo de médicos estudiados resultó ser de nivel medio bajo. No se encontraron diferencias atribuibles al sexo (Tabla 3 ).

\section{Habilidades diagnósticas (HD)}

Se evaluó a través de dos dimensiones: "Reconocimiento de síntomas" (RS) y "Clasificación de síntomas" (CS). Los MGs obtuvieron mejores resultados en RS ( $\operatorname{Min}=40,82 ; \operatorname{Max}=87,07)$ que en CS. En la dimensión RS, sólo se observaron diferencias significativas entre médicos chilenos y extranjeros. Los primeros tienden a reconocer más apropiadamente los síntomas mostrando $36,7 \%$ de mejores puntajes (Tabla 4). No hubo diferencias significativas en relación con el sexo ni la edad.

Tabla 3. Diferencias de medias (índice) en conocimientos teóricos para el manejo de la depresión por médicos generales de APS de RM. Santiago, Chile

\begin{tabular}{|llllllc|}
\hline Variable & Categoría & n & \multicolumn{2}{c}{ Promedio (SD) } & t & Cohen's d \\
Sexo & Mujer & 38 & 39,1 & $(11,11)$ & & 0,05 \\
& Hombre & 18 & 38,5 & $(12,45)$ & 0,171 & 0,48 \\
Edad & Adulto joven & 30 & 41,4 & $(11,18)$ & & $1,789^{*}$ \\
& Adulto & 26 & 36,0 & $(11,28)$ & & $-0,46$ \\
\hline
\end{tabular}

*Significativo a $90 \%$ de confianza $(p<0,10)$.

Tabla 4. Diferencias de medianas de reconocimiento de síntomas para el manejo de la depresión por médicos generales de APS de RM. Santiago, Chile

\begin{tabular}{|lllccc|}
\hline Variable & Categoría & $\mathbf{n}$ & Puntaje medio & Za & A (tamaño efecto) \\
Sexo & Mujer & 38 & 27,6 & & 0,315 \\
& Hombre & 18 & 30,2 & $-0,544$ & 0,544 \\
Edad & Adulto joven & 30 & 29,6 & & $-0,559$ \\
\multirow{2}{*}{ Nacionalidad } & Adulto & 26 & 27,1 & & 0,367 \\
& Extranjero & 32 & 25,3 & $-1,698^{*}$ & \\
\hline
\end{tabular}

aTransformación a puntuación Z del estadístico U de Mann-Whitney. *Significativo a 90\% de confianza $(p<0,10)$. 
Tabla 5. Distribución de habilidades de clasificación de síntomas (índice) para el manejo de la depresión por médicos generales de APS de RM. Santiago, Chile

\begin{tabular}{|ccccccc|}
\hline \% Respuestas correctas & $\mathbf{0}$ & $\mathbf{2 5}$ & $\mathbf{5 0}$ & $\mathbf{7 5}$ & $\mathbf{1 0 0}$ & Total \\
\hline$\% \mathrm{MG}$ & 46,4 & 41,1 & 8,9 & 3,6 & 0 & 100 \\
\hline $\mathrm{n}$ & 26 & 23 & 5 & 2 & 0 & 56 \\
\hline
\end{tabular}

Los MGs mostraron niveles muy bajos en la dimensión CS. Casi la mitad de ellos no respondió ninguna pregunta correcta, como tampoco ninguno respondió correctamente las cuatro preguntas (Tabla 5).

Dado lo anterior, se hizo un segundo análisis con datos recodificados mediante dicotomización: médicos con ninguna respuesta correcta $(\mathrm{n}=26)$ y médicos con una o más respuestas correctas $(\mathrm{n}=30)$, pese a lo cual no hubo asociaciones estadísticamente significativas en ninguna variable. No se observaron diferencias en CS entre los MGs participantes del estudio para ninguna de las variables estudiadas (Tabla 5).

\section{Habilidades terapéuticas (HT)}

La muestra de médicos presentó niveles de habilidades terapéuticas que van desde $0 \%$ a $67 \%$ de aciertos en el test, con una mediana de 33\%. Sólo 14,3\% de los MGs logró acertar a 50\% o más de los ítems que miden HT.

No se encontraron diferencias significativas en HT para ninguna de las variables estudiadas; lo mismo que en CT, la tendencia en puntajes observados fue a concentrarse en promedios por debajo de 50\% del índice. Es decir, las HT también resultaron de nivel medio bajo para todo el grupo de MGs participantes (Tabla 6).

\section{Discusión}

El presente estudio entrega una evaluación de los niveles de CT, HD y HT para el manejo de la depresión en una muestra voluntaria de MGs de Centros de Salud Primaria de la Región Metropolitana. Si bien no se trata de una muestra aleatoriamente seleccionada del universo de MGs que trabajan en la RM, en ella están representados los dos grandes grupos de médicos que constituyen la fuerza laboral habitual en estos centros: médicos chilenos y extranjeros ${ }^{39}$. Destacamos por tanto, la

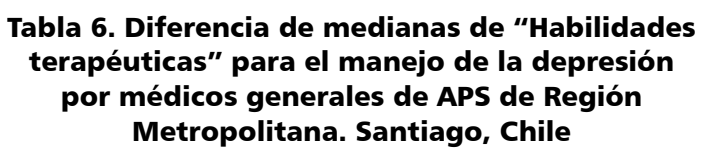

\begin{tabular}{|lllcc|}
\hline Variable & Categoría & $\mathbf{n}$ & $\mathbf{Z}^{*}$ & $\begin{array}{c}\text { A (tamaño } \\
\text { efecto) }\end{array}$ \\
\hline Sexo & Mujer & 38 & & \\
& Hombre & 18 & $-1,40$ & 0,62 \\
Edad & Adulto joven & 30 & & \\
& Adulto & 26 & $-0,75$ & 0,56 \\
Nacionalidad & Extranjero & 32 & $-1,16$ & 0,41 \\
& Chileno & 24 & -16 & \\
\hline
\end{tabular}

*Transformación a puntuación $Z$ del estadístico $U$ de Mann-Whitney.

importancia de conocer en detalle los niveles de habilidades clínicas que disponen estos MGs en el manejo de la depresión, una patología de alta prevalencia en nuestro país.

Cabe destacar el bajo nivel de CT observado en la muestra, encontrándose todos los MGs con índices bajo 50\%. Médicos más jóvenes y chilenos mostraron mejores niveles de CT de depresión que médicos adultos y extranjeros. La diferencia en CT mostrada por los médicos jóvenes podría explicarse por la proximidad temporal con los estudios de pregrado, en tanto que la diferencia entre MGs chilenos y extranjeros, podría atribuirse a los programas de formación de cada país, dado que en Chile se han reformulado de acuerdo a las patologías más prevalentes e incorporadas en el plan AUGE, entre ellas la depresión. En otros países la formación podría tener énfasis en otras patologías más prevalentes ${ }^{38}$. Estos factores podrían explicar parte de las diferencias observadas en CT entre médicos chilenos y extranjeros, lo que coincide con los resultados generales del Examen Único Nacional de Conocimientos de Medicina que incluye estos temas (EUNACOM ${ }^{40}$.

En general, los MGs mostraron bajos niveles 
en HD y HT, lo cual podría responder a la mayor complejidad de estas destrezas, aspecto que ha sido considerado en la elaboración de los programas de pregrado de distintas universidades en Chile, particularmente a través del internado de psiquiatría obligatorio $^{31,41}$. Pese a lo anterior, se considera que aún se requiere de entrenamiento de postítulo en esta materia ${ }^{35,36}$.

Asimismo se podría plantear que estos resultados reflejan una medición con un nivel de exigencia relativamente alto, ya que al tratarse de habilidades complejas se necesitan métodos de evaluación también complejos que incluyan varias mediciones y formatos diferentes ${ }^{26,27,30,35,36}$. Por ello un desafío importante del estudio consistió en la elaboración de la metodología de evaluación que buscó dar cuenta del razonamiento clínico en aspectos diagnósticos y terapéuticos, como parte de la competencia clínica ${ }^{26}$.

Los mejores resultados observados en RS podrían atribuirse a habilidades diferentes a las evaluadas en este estudio, tales como habilidades relacionales, comunicacionales y hábitos mentales, las cuales formarían parte de las estrategias usadas en la formulación del diagnóstico de depresión ${ }^{25}$.

Las diferencias significativas en RS entre MGs chilenos y extranjeros podrían atribuirse a diferencias culturales, dado que los MGs extranjeros realizan el ejercicio diagnóstico a partir de un marco cultural diferente al de los pacientes $y$, por lo tanto, el significado de los síntomas es distinto $^{42}$. Esto resulta relevante en el diagnóstico de la depresión, pues los MGs reconocen problemas para diferenciar estados depresivos de reacciones a problemas sociales ${ }^{25}$. Además, la mayor frecuencia de observación de una patología, en tanto fuente de aprendizaje práctico, resulta un facilitador del buen diagnóstico de depresión por parte de médicos chilenos ${ }^{38,43}$. Se destaca este hallazgo pues dada la alta prevalencia de depresión en Chile, sería recomendable que los MGs extranjeros participasen de un proceso de inducción que favorezca su desempeño clínico en esta materia, tal como se sugiere en otras realidades ${ }^{42,44}$.

Las limitaciones del estudio podrían derivar del tipo de procedimiento de muestreo donde la motivación sería un factor importante en el reclutamiento de participantes; esto puede haber introducido sesgos por haber captado a quienes, conociendo sus carencias en materia de psiquiatría, se interesaron y participaron en el estudio, lo cual puede explicar la tendencia a observar bajos niveles en las habilidades clínicas evaluadas.

Asimismo, debido al tamaño muestral con el que se pudo contar para el presente estudio, no fue posible realizar análisis que permitieran identificar perfiles más precisos de los MGs en función de sus conocimientos, habilidades y características sociodemográficas. Esperamos que futuros estudios con tamaños muestrales más amplios permitan establecer dichos perfiles.

Otra limitación se relacionaría con la metodología de evaluación de las habilidades clínicas, las cuales debieron adecuarse a las restricciones propias del estudio relativas a recursos técnicos y financieros, quedando pendiente para futuros estudios la evaluación de desempeños clínicos directos. No obstante, este trabajo entrega una valiosa información por tratarse del primer estudio de este tipo a nivel nacional.

Por último, es importante considerar la evaluación de habilidades clínicas como un indicador de la calidad con que se desarrolla la atención en salud mental, con el objeto de identificar déficits posibles de mejorar. Los resultados de este estudio debieran promover la revisión de las metodologías de enseñanza-aprendizaje en materias de salud mental y psiquiatría.

\section{Referencias}

1. Araya R, Rojas G, Fritsch R, Acuña J, Lewis G. Common mental disorders in Santiago, Chile: prevalence and sociodemographics correlations. Brit J Psychiat 2001; 178: 228-33.

2. Florenzano R, Acuña J, Fullerton C, Castro C. Estudio comparativo de frecuencia y características de los trastornos emocionales en pacientes que consultan en el nivel primario de atención en Santiago de Chile. Rev Med Chile 1998; 126: 397-405.

3. Florenzano R, Acuña J, Fullerton C, Castro C. Results from the Santiago the Chile Center. En: Üstün TB y Sartorius N, Editores, Mental Illness in General Health Care: WHO international study. Chichester, England, Editorial John Wiley \& Sons; 1995: 247-63.

4. Goldberg DP, Lecubrier Y. Form and frequency of mental disorders across centres. En: Üstün TB y Sartorius $\mathrm{N}$, Editores, Mental illness in general health care: an international study. Chichester, England, Editorial John Wiley \& Sons; 1995: 323-34.

5. Vicente B, Khon R, Rioseco P, Saldivia S, Levav I, Torres 
S. Lifetime and 12-month prevalence of DSM-III-R disorders in the Chile psychiatric prevalence study. Am J Psych 2006; 163 (8): 1362-70.

6. Vicente B, Kohn R, Rioseco P, Saldivia S, Baker Ch, Torres S. Population prevalence of psychiatric disorders in Chile: 6-month and 1 month rates. Br J Psychiat 2004; 184: 299-305.

7. Minnoletti A, Zaccaria A. Plan Nacional de Salud Mental en Chile: 10 años de experiencia. Rev Panam Salud Publ Am J Public Health 2005; 18 (4/5): 346-58.

8. Programa nacional de diagnóstico y tratamiento de la Depresión. MINSAL. Chile. Disponible en: www.minsal. cl [Consultado el 14 de mayo de 2014].

9. Alvarado R, Vega J, Sanhueza G, Muñoz M. Evaluación del Programa para la Detección, Diagnóstico y Tratamiento Integral de la Depresión en atención primaria, en Chile. Rev Panam Salud Publ 2005; 18: 278-86.

10. Guía Clínica AUGE Depresión. 2013. MINSAL. Chile. Disponible en: www.minsal.cl [Consultado el 14 de mayo de 2014].

11. Alvarado R, Rojas G. El programa nacional para el diagnóstico y tratamiento de depresión en atención primaria: una evaluación necesaria. Rev Med Chile 2011; 139: 592-9.

12. World Health Organization (2001). The World Health Report 2001: Mental health: New Understanding, New Hope. Geneva, WHO; 2001.

13. OMS. WHO. Organization of services for mental health. Geneva: WHO; 2003.

14. World Mental Health. Survey Consortium. Prevalence, severity and unmet need for treatment of mental disorders in the World Health Organization, World health survey. JAMA 2004; 291 (21): 2581-90.

15. Kohn R, Saxena S, Levav I, Saraceno B. The treatment gap in mental health care. B World Health Organ 2004; 82 (11): 858-66.

16. Faulkner A, Mills N, Bainton D, Baxter K, Kinnersley $\mathrm{P}$, Peters TJ, et al. A systematic review of the effect of primary care-based service innovations on quality and patterns of referral to specialist secondary care. Br J Gen Pract 2003; 878-84.

17. Cepoiu M, McCusker J, Cole M, Sewitch M, Belzile E, Ciampi A. Recognition of depression by non-pychiatric physicians: a systematic literature review and meta-analysis. J Gen Intern Med 2008; 23 (1): 25-36.

18. Beth P, Hugtenburg J, Penningx B, van Balkom A, Nolen $\mathrm{W}$, Hoogendijk. Treatment inadequacy in primary and specialized care patients with depressive and/or anxiety disorders. Psychiatry Res 2013; 210 (2): 594-600.

19. Levav I, Kohn R, Montoya I, et al. Training Latin American Primary care physicians in the WPA module on depression: results of a multicenter trial. Psychol Med 2005; 35 (1): 35-45.

20. Kohn R, Levav I, Caldas de Almeida JM, Vicente B, Andrade L, Caraveo-Anduaga JJ, et al. Los trastornos mentales en América Latina y el Caribe: asunto prioritario para la salud pública. Rev Panam Salud Pub 2005; 18 (4/5): 229-40.

21. Vicente B, Kohn R, Levav I, Espejo F, Saldivia S, Sartorius $\mathrm{N}$. Training primary care physicians in Chile in the diagnosis and treatment of depression. J Affect Disord 2007; 98 (1-2): 121-7.

22. Thompson C, Kinmonth AL, Stevens L, Pevele RC, Stevens A, Ostier KJ, et al. Effects of a clinical-practice guideline and practice-based education on detection and outcome of depression in primary care: Hampshire Depression Project randomized controlled trial. The Lancet 2000; 355: p. 185-91.

23. Hodge B, Inch C, Silver I. Improving the psychiatric knowledge, skills, and attitudes of primary care physicians, 1950-2000: A review. Am J Psychiatry 2001; 158: 1579-86.

24. Barley E, Murray J, Walters P, Tylee A. Managing depression in primary care: A meta-synthesis of qualitative and quantitative research from UK to identify barriers and facilitators. Disponible en: www.biomedcentral.com [Consultado el 14 de mayo de 2014].

25. Schumann I, Schneider A, Kantert C, Löwe B, Linde K. Physician's attitudes, diagnostic process and barriers regarding depression diagnosis in primary care: a systematic review of qualitative studies. Fam Pract 2012; 29: 255-63.

26. Epstein R, Hundert E. Defining and assessing professional competence. JAMA 2002; 287 (2): 226-35.

27. Rajasoorya C. 9th College of Physicians Lecture: Medical education and Professional Training-Changing the Trajectory. Annals Academy of Medicine 2013; 42 (2): 99-104.

28. Beckman H, Wendland M, Mooney Ch, Krasner M, Quill T, Suchman A, et al. The impact of a program in mindful communication on primary care physicians. Acad Med 2012; 87 (6): 1-5.

29. Miller G. The Assessment of Clinical Skills/Competence/ Performance. Acad Med 1990; 656 (9): 563-7.

30. Epstein R. Assessment in medical education. N England J Med 2007: 356 (4): 387-96.

31. Tala A, Rojas G, Marín C. Evolución de la enseñanza de Psiquiatría en el pregrado de medicina: una revisión de 35 años. Rev Psiq Clín 2012; 50 (2): 27-34.

32. Herskovic P. La reforma curricular de la Escuela de Medicina de la Universidad de Chile. Rev Chil Pediatr 2005; 76: 9-11. 
33. Rosselot E. Planificando la educación médica para las próximas décadas. Rev Med Chile 2001; 129: 1473-8.

34. Millán T, Ercolano M, Pérez M, Fuentes C. Autoevaluación de habilidades clínicas básicas en médicos recién egresados de la Facultad de Medicina, Universidad de Chile. Rev Med Chile 2007; 135: 1479-86.

35. Leigh H, Stewart D, Mallois R. Mental health and psychiatry training in primary care residency programs. Part I. Who teaches, where, when and how satisfied? Gen Hosp Psychiat 2006; 28: 189-94.

36. Leigh H, Stewart D, Mallois R. Mental health and psychiatry training in primary care residency programs. Part II. What skills and diagnoses are taught, how adequate, and what affects training directors' satisfaction? Gen Hosp Psychiat 2006; 28: 195-204.

37. ACGME (2000) Outcome Project. Accreditation Council for Graduate Medical Education Web site. Disponible en: www.acgme.org [Consultado el 1 de abril de 2012].

38. Huepe G, Cárcamo M, Acuña J, Botto A, Jiménez JP. (2014). Impacto de una Capacitación en Trastornos Depresivos para Médicos Generales de Atención Primaria en Salud. Resultados Cualitativos [Aceptado para publicación el 20 de abril de 2015 en Revista Médica de Chile].

39. Memoria de la Agrupación Nacional de Médicos de Atención Primaria (julio de 2010-junio de 2012) p. 3738. Disponible en: www.medicosaps.cl [Consultado el 16 de mayo de 2014].

40. Emol. 91\% de médicos titulados en el extranjero reprueba test Eunacom [Noticia publicada el 13 de enero de 2014]. Disponible en: www.emol.com [Consultado el 5 de marzo de 2014].

41. Valdivieso S, Sirhan M, Aguirre C, Ivelic J, Aillach E, Villarroel L. Attitudes of Medical Students Toward Psychiatry in a Chilean Medical School. Acad Phychiatry 2014; 38: 309-11.

42. Weissman J, Betancourt J, Campbell E, Park E, Kim M, Clarridge B, et al. Resident Physicians' Preparedness to Provide Cross-Cultural Care. JAMA 2005; 294 (9): 105867.

43. Kleiman A. Culture and depression. N Engl J Med 2004; 351 (10): 951-3.

45. Betancourt J. Cultural competence-marginal or mainstream movement? N Engl J Med 2004; 351 (10): 953-5. 